\section{Pilot Study Demonstrating that Immunostimulation with $\beta$ glucan Fails to Moderate the Immunosup- pressive Effects of Dexamethasone when Co-administered to Rainbow Trout Oncorhynchus mykiss Experimentally Challenged with the Microsporidian Loma salmonae}

\author{
David J. Speare*, Nicole J. Guselle \\ and Jennifer E. Harkness \\ Department of Pathology \& Microbiology, Atlantic \\ Veterinary College, PE, CIA 4P3, Canada
}

(Received June 4, 2011)

\begin{abstract}
In this pilot study, juvenile rainbow trout were experimentally challenged with the microsporidian Loma salmonae to determine the effects of immunostimulation with $\beta-1,3 / 1,6$ glucan administered in the feed at $200 \mathrm{mg} / \mathrm{kg}$ of feed, and immunosuppression with dexamethasone at 300 $\mathrm{mg} / \mathrm{kg}$ of feed when these were administered in the early stages of infection. Six weeks after oral exposure to Loma spores, control fish developed a mean of 34.8 spore filled xenomas per gill arch; those which had received $\beta$ glucan had significantly fewer xenomas (23.6) whereas those receiving dexamethasone had significantly more (43.0). Paradoxically, when fish received both $\beta$ glucan and dexamethasone in any of three temporal combinations, xenoma numbers were significantly higher (51.0-65.1) even when compared to fish receiving only dexamethasone.
\end{abstract}

Key words: microsporidia, Loma salmonae, dexamethasone, beta-glucan, immunosuppression

The microsporidian Loma salmonae, the causative agent of Microsporidial Gill Disease of Salmon (MGDS), is an endemic seasonal cause of morbidity and mortality affecting farmed Pacific salmon particularly along Canada's west coast since its first report in 19891). The disease has been extensively modelled and studied in rainbow trout 0 . mykiss ${ }^{2)}$; through this model, previous work has demonstrated and partially characterized host innate and acquired immunity ${ }^{3)}$. Innate immunity, the response of the host to first encountering $L$. salmonae, has been shown to be inhibited by physiological stress

\footnotetext{
* Corresponding author

E-mail: speare@upei.ca
}

as modelled through the effects of dexamethasone administration to trout ${ }^{4}$. In contrast, immunostimulation as modelled through the effects of $\beta-1,3 / 1,6$ glucan can markedly boost innate immunity against $L$. salmonae ${ }^{5}$.

Within an aquaculture setting, disease outbreaks often follow events that trigger physiological stress. For example, disease outbreaks in marine netpens of salmon often follow handling (grading of fish), the presence of divers in netpens, severe storms, the presence of predators such as seals, or concurrent diseases including effects of algal blooms. Furthermore, with the development of integrated multitrophic aquaculture approaches to marine culture of salmon, a practice growing in popularity whereby several species of aquatic life are cultured on salmon farm leases thereby capitalizing on various nutrients flowing from caged fish, it is possible that farmed salmon will be more frequently disturbed by human activities related to maintenance of diverse aquaculture infrastructures and harvest of products from various trophic levels. Many of these routine activities, which can be inadvertently but unavoidably stressful to fish, can at least be scheduled; it may therefore be possible to minimize the effect of stress on fish, particularly the effect of stress on innate resistance to disease, through the scheduled prophylactic or metaphylactic application of an immunostimulant either before, during, or following a stressful event. The purpose of the current pilot study is to determine the degree to which immunostimulation of trout, with an effective level of orally administered $\beta$ glucan $(\beta-1,3 / 1,6)$, can ameliorate the effects of orally administered dexamethasone as assessed by the number of xenomas forming on the gills of fish during an experimental model of MGDS. Exposure periods of the two treatments, relative to the exposure time of fish to $L$. salmonae, where chosen in order to evaluate the relative value of providing an infeed immunostimulant either during or after a stressful event, herein modelled through dexamethasone exposure.

From a group of 2000 hatchery derived rainbow trout, from a facility with no history of $L$. salmonae infections, 360 fish (15-20 g) were arbitrarily selected and randomly allocated into each of six $70 \mathrm{~L}$ fibreglass tanks with 60 fish per tank. All tanks received the same water supply from a header tank; flow rates of $2 \mathrm{~L} \mathrm{~min}^{-1}$ were used and water temperature was $15.0^{\circ} \mathrm{C}$. Fish were fed a 2-mm extruded pellet (HiPro, Corey Aquafeeds) 3 times per week at a rate equal to $2 \%$ of fish biomass. Two weeks prior to fish being challenged with $L$. salmonae, the following in-feed treatment protocols were allocated to tanks. Fish in Tank A received feed which had been gelatin coated such that the feed contained $300 \mathrm{mg}$ of dexamethasone (Dexasone, Valeant) ${ }^{4)}$ and $200 \mathrm{mg}$ of $\beta$ glucan

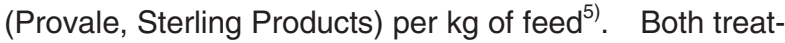
ments ended at the time of Loma challenge. Fish in 
Tank $B$ received dexamethasone treatment prior to Loma challenge, and then received only $\beta$ glucan treatment for two weeks after Loma challenge. Fish in Tank $\mathrm{C}$ received $\beta$ glucan prior to Loma challenge, and then received only dexamethasone for two weeks after Loma challenge. Fish in Tank $D$ received dexamethasone treatment prior to Loma challenge and did not receive $\beta$ glucan whereas fish in Tank $\mathrm{E}$ received $\beta$ glucan treatment prior to Loma challenge but did not receive dexamethasone. Tank F, serving as the positive control and standard, did not receive either dexamethasone or $\beta$ glucan prior to or after Loma challenge. Fish from the initial holding tank were assessed periodically to ensure that $L$. salmonae was not present in the research facility at a background level.

Loma challenge was as previously published ${ }^{5)}$; the procedure involved harvesting of xenoma-bearing gill tissues from trout previously infected with L. salmonae. Gills were finely macerated and equal amounts were introduced to each of the 6 tanks; fish were observed to ensure that all gill material was consumed. Six weeks after exposure to gill material, all of the fish were euthanized with an overdose of benzocaine and the left branchial arch dissected free, prepared as a whole-mount for light microscopy, and the number of xenomas was determined. Data was expressed as 'xenoma counts per gill arch' (XCPGA); using this, the mean values for each tank were calculated and reported herein (Table 1). GraphPad Prism Version 2 (GraphPad Inc.) was used for statistical comparison between groups and a $P$-value of 0.05 or less was considered significant. NewmanKeuls post tests for specific parametric comparisons followed ANOVA assessment. Median values were assessed with the Kruskal-Wallis test followed by Dunn's test for specific non-parametric comparisons.

During the trial, it was observed that all of the offered feed was consumed at each meal; additionally, when macerated gill tissue was introduced, it was all

Table 1. Mean xenoma counts per gill arch (XCPGA) and standard error of the mean (SEM) in groups of fish exposed to combinations of beta glucan as Pro Vale (PV) and dexamethasone (dex) either two weeks before or two weeks after experimental exposure (exp.) to L. salmonae. XCPGA values in Tanks D \& $E$ are significantly different from that in tank $F$; XCPGA values in Tanks $A-C$ are significantly different from that in Tank D

\begin{tabular}{lccc}
\hline \multicolumn{1}{c}{ Tank Letter \& Treatment Group } & N & $\begin{array}{c}\text { Mean } \\
\text { XCPGA }\end{array}$ & SEM \\
\hline Tank A: PV and Dex before exp. & 60 & 63.5 & 3.30 \\
Tank B: Dex before \& PV after exp. & 60 & 51.0 & 2.30 \\
Tank C: PV before \& Dex after exp. & 60 & 65.1 & 3.50 \\
Tank D: Dex before exp. & 60 & 43.0 & 1.30 \\
Tank E: PV before exp. & 60 & 23.6 & 1.90 \\
Tank F: No treatment & 60 & 34.8 & 1.35 \\
\hline
\end{tabular}

quickly consumed. During the trial there were no mortalities in any of the groups. By week 6, well-formed xenomas, typical for MGDS were found distributed randomly throughout the entire gill tissue; there was no evidence that xenomas had already begun to rupture. Results are detailed in Table 1. In the control group (Tank F), the mean XCPGA was 34.8. In contrast, the mean XCPGA in Tank E ( $\beta$ glucan only) was reduced by $32 \%$ to 23.6 ; compared to the control group the mean XCPGA in Tank D (dexamethasone only) was increased by $23 \%$ to 43.0 . Both of these changes were statistically significant. Based on results in the control tank, and compared to previous literature from our MGDS model ${ }^{2)}$, we conclude that the infection trial was successful and led to an XCPGA level typical of moderately -severe infections. The in-feed administration of $\beta$ glucan provided the desired effect as defined within previous studies ${ }^{5}$; its use led to a significant level of protection based on the XCPGA reduction in treated fish. The in-feed administration of dexamethasone also produced the desired effect by creating a significantly elevated XCPGA in treated fish. As a critique of our model of immunosuppression/immunostimulation, the doses that we used appear reasonable in that the effects of either treatment were approximately the same, although in opposite directions. Since neither dexamethasone nor $\beta$ glucan are known to have a direct anti-infective effect on microsporidia, and since our use pattern of treatments and pathogen exposure method did not provide the time-frame for an acquired immune response to be elicited which would influence the numbers of xenomas arising from the initial experimental exposure in these otherwise naive fish ${ }^{3)}$, we conclude that the actions of both in-feed treatments are likely mediated through modulation of the innate defence system.

In Tanks A, B and C (representing temporal variations of the combinations of dexamethasone and $\beta$ glucan) the mean XCPGA values were 63.5, 51.0 and 65.1 respectively; compared to values in the control tank these values represent increases of $82 \%, 46 \%$, and $87 \%$ respectively; all of these differences were statistically significant when compared to the control tank (Tank F) of fish. Furthermore, fish receiving dexamethasone/ $\beta$ glucan treatments had XCPGA values which were higher than for fish receiving dexamethasone alone; this difference was statistically significant when outcomes in Tank A, Tank B or Tank C were compared to Tank D.

Results in the current study support previous demonstrations of the practical value of orally administered $\beta$ glucan against $L$. salmonae ${ }^{5)}$, additionally, our results support previous work in which dexamethasone has been shown to render trout more susceptible to Loma challenges ${ }^{4}$; the current study also shows that $\beta$ glucan administration, when delivered at a level shown to be 
effective in stimulating innate immunity, was nonetheless unable to antagonize the immunosuppressive effects of dexamethasone. Indeed, paradoxically, $\beta$ glucan's effect appeared to be additive to those of dexamethasone when the two agents were used in combination. The effect is not predicted, nor easy to explain based on what is currently known about the relevant cell-signalling and expression pathways. Nonetheless, it suggests that further research is needed to determine whether, in general, $\beta$ glucan administration should be withheld prior to periods of anticipated fish stress, and after events which are suspected to have caused fish stress. Our study is preliminary in that we only examined a specific dose of $\beta$ glucan and a specific level of immunosuppression as would arise from the particular dose of dexamethasone. To provide a mechanistic underpinning, work to better understand the cross-talk between immunostimulants and immunosuppressants is clearly warranted given the unexpected nature of the results demonstrated in this pilot study.

\section{Acknowledgements}

For relevant aspects of this work, we greatly appreciate the support received from the Natural Sciences and Engineering Research Council of Canada (NSERC) Strategic Canadian Multi-Trophic Aquaculture Network (CIMTAN) in collaboration with its partners, Fisheries and Oceans Canada, The University of New Brunswick, Cooke Aquaculture Inc., Kyoquot SEAfoods Ltd. and Marine Harvest Canada Ltd.

\section{References}

1) Speare, D. J., J. Brackett and H. W. Ferguson (1989): Can. Vet. J., 30, 571-575. 2) Becker, J. A. and D. J. Speare (2007): An. Hith. Res. Rev., 8, 1-10. 3 3) Rodriguez-Tovar, L. E., D. J. Speare and R. J. F. Markham (2011): Fish Shellfish Immunol., 30, 999-1006. 4) Lovy, J., D. J. Speare, H. Stryhn and G. M. Wright (2008): Fish Shellfish Immunol., 24, 649-658. 5) Guselle, N. J., D. J. Speare, R. J. F. Markham and S. Patelakis (2010): North Am. J. Aquacult., 72, 65-72. 